\title{
PHOTOACOUSTIC STUDY OF THE INTERACTION BETWEEN THIN OIL LAYERS WITH WATER
}

\author{
J. SzUrkowski ${ }^{a}$, I. PAWELSKa ${ }^{a}$, S. WARTeWIG ${ }^{b}$ AND S. Pogorzelski ${ }^{a}$ \\ ${ }^{a}$ Institute of Experimental Physics, University of Gdanisk \\ Wita Stwosza 57, 80-952 Gdańsk, Poland \\ ${ }^{b}$ Martin-Luther-Universität Halle-Wittenberg \\ Fachbereich Pharmazie, Hoher Weg 7, 06099 Halle, Germany
}

(Received November 30, 1999; revised version March 24, 2000)

In the presented paper the structural changes in thin olive oil layers taking place as a result of interaction with water using a photoacoustic technique were studied. The oil layers were spread from a solution in a volatile solvent (ethyl ether) on the water and copper surface. These studies are a natural continuation of the previous work on a layered system performed within the light range $(680 \mathrm{~nm})$, which pointed to the irregularities of oil layers related to their thickness is now expanded to the infrared band. A structural irregularity was observed for a layer thickness of $100 \mu \mathrm{m}$, but it did not appear for the one of pure bulk oil. Signatures of irregularity were clearly observed in the phase plots whereas the amplitude dependences were not sensitive to them. The performed studies allowed one to relate the diversity of thermal parameters in the samples with structural changes found in oil layers originated from oil-water interactions.

PACS numbers: $81.70 . \mathrm{Cv}, 36.20 . \mathrm{Ng}, 68.15 .+\mathrm{e}$

\section{Introduction}

Several processes of great scientific and technological impact are governed by surfactant structure and its monolayer properties in liquid mixtures particularly at interfaces. The viscoelastic properties and adsorption kinetics of specific constituents at fluid-fluid interfaces often play a major role in determining the final result. Thus modelling of the monolayer morphology represents a necessary step to fully understand and improve such processes as food production, oil recovery, lubrication, stabilisation of emulsions, chemical engineering, and production of pharmaceuticals or cosmetics [1]. In natural systems, the air-sea exchange of organic and inorganic matter, gases, water vapour, heat, and momentum are affected by the adsorptive surfactant layer. 
As a model organic substance, olive oil was selected to the studies of layered oil-water and oil-solid substrate systems. Natural olive oil contains a wide variety of components like triglycerides, diglycerides, monoglycerides, fatty acid, and alcohol (glycerol) [2]. Physical and chemical properties of glycerides have been studied for more than a century. Based on X-ray technique and Raman spectroscopy, a proposed structure of triglycerides in the liquid state could be found in [3]. The hydrocarbon chains are disordered and the proportion of gauche conformation is large. The triglycerides are arranged in a chair-shaped conformation. The proposed model is dynamic; that is, both the size and orientation of the lamellar units vary with diffusion rates of the molecules. A degree of order within the lamellar units is proposed to be almost constant.

So far, measurements performed with a classic photoacoustic method (so-called thermal depth profiling) clearly exhibited signatures characteristic of the interaction of thin olive oil layers with the underlying water subphase [4-7]. The results obtained point to the presence of a distinguishable 3-layer structure for oil layers ranging from 50 to $100 \mu \mathrm{m}$ in thickness if spread on water. At a depth of about 9 to $13 \mu \mathrm{m}$, a $4 \mu \mathrm{m}$ thick layer of different thermal properties than the surrounding oil was found.

In our work the analysis of the infrared spectra in the band characteristic of $\mathrm{CH}$ atomic bands of olive oil was of particular interest. We tried to separate symmetric and asymmetric vibration modes of the functional groups [4]. It is standard in vibration spectroscopic studies on lipids to take the intensity ratio of the asymmetric to the symmetric $\mathrm{CH}_{2}$ stretching modes as a measure of the relative population of the trans and gauche conformers and with it as a degree of order of the alkyl chains [8]. Moreover, a degree of order increases with growing asymmetric vibrations in the considered system.

\section{Material and methods}

Olive oil was bought from the local market and used without further purification. In the studied model system thin layers of olive oil (ranging from 12.5 to $250 \mu \mathrm{m}$ in thickness) were dissolved in ethyl ether and then spread onto the clean water and copper surfaces.

In measurements we have taken advantage of two (supplementary to each other) techniques of the photoacoustic (PA) signal measurement. One of them, the classical photoacoustic method (one beam steady state photoacoustic spectrometer in which the signal is evaluated by means of the phase-sensitive method realized using a lock-in amplifier), is based on a laser diode as a light source which allows a precise adjustment of the light beam modulation frequency with a very fine step, and subsequently performing the sample depth profiling with a high accuracy. In the photoacoustic spectroscopy (PAS) measurements the thermal diffusion length $\mu_{\mathrm{s}}$ decreases with an increase in the modulation frequency $f$. The sampling depth (thermal diffusion length) is given by [4]

$$
\mu_{\mathrm{s}}(f)=\sqrt{\frac{\beta_{\mathrm{s}}}{\pi f}},
$$


where $\beta_{\mathrm{s}}=k_{\mathrm{s}} / \rho_{\mathrm{s}} c_{\mathrm{s}}-$ thermal diffusivity $\left[\mathrm{m}^{2} / \mathrm{s}\right], k_{\mathrm{s}}$ - thermal conductivity $[\mathrm{J} /(\mathrm{s} \cdot \mathrm{m} \cdot \mathrm{K})], c_{\mathrm{s}}$ - heat capacity $[\mathrm{J} /(\mathrm{kg} \cdot \mathrm{K})], \rho_{\mathrm{s}}$ - density $\left[\mathrm{kg} / \mathrm{m}^{3}\right], f$ - frequency of light modulation $[\mathrm{Hz}]$.

The aim of the measurements performed with a classic photoacoustic technique was to follow the thermal changes (irregularities) in thin olive oil layers deposited on the water and copper surfaces. An amplitude and phase of the photoacoustic signal were measured within a wide range of light modulation frequencies, ranging from $1 \mathrm{~Hz}$ to $1 \mathrm{kHz}$, using a classic photoacoustic spectrometer. The signal amplitude and phase dependences are presented as a function of reciprocal of square root of the modulation frequency $\left(f^{-1 / 2}\right)$ in the range of $0.03-0.37 \mathrm{~Hz}^{-1 / 2}$. In such a scaling presentation, there is a linear dependence between the modulation frequency and penetration depth of the photoacoustic signal where a proportionality factor is expressed by the thermal parameters of the studied sample according to Eq. (1). A thermal diffusion length is strongly dependent on the light modulation frequency. Knowing the values of the thermal properties of oil and water in contact, the depth from which the signal comes from can be determined.

In the presented studies a setup based on a lock-in amplifier SR 850 was used, which not only measured the signal but also controlled an operation of the laser diode being a source of modulated light. A block diagram of the setup is presented in Fig. 1. A laser diode (type SDL-73311) with a wavelength of $680 \mathrm{~nm}$ was a light source for olive oil spread on the water surface and a laser diode (type SDL-5311-G1) of $800 \mathrm{~nm}$ for olive oil spread on the copper surface. The laser beam is provided to the measuring cell of the author's construction by a fiber wire $[4,6]$.

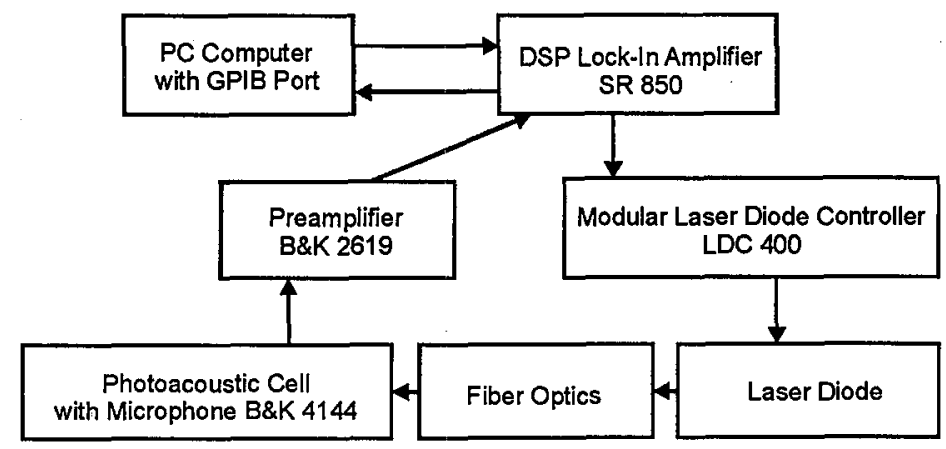

Fig. 1. Block diagram of the laser diode light modulation PAS system.

The second technique used here - the Fourier transform infrared photoacoustic spectroscopy (FTIR PAS) - applies only a limited set of modulation frequencies. However, the selection of infrared region gives an opportunity to obtain the photoacoustic spectra in wavelength bands characteristic of absorption of chemical bonds of molecules.

The photoacoustic spectra in the infrared region were measured using a Bruker FT-IR JFS 28 spectrometer equipped with a MTEC model 200 photoacoustic cell from the Department of Pharmacy, Martin-Luther-University, Halle, Germany. 


\section{Results and discussion}

The measurements performed pointed to differences in homogeneity of thin olive oil layers spread over the water surface [4-7]. In order to finally decide whether the layered structures in oil result from the oil-supporting water interaction or originate from the method of oil layer spreading, the photoacoustic studies were performed on olive oil samples deposited onto a metallic copper surface.

The photoacoustic signal amplitude and phase plots, for a $100 \mu \mathrm{m}$ olive oil layer spread on the water $(a, b)$ and copper $(c, d)$ surfaces is shown in Fig. 2.

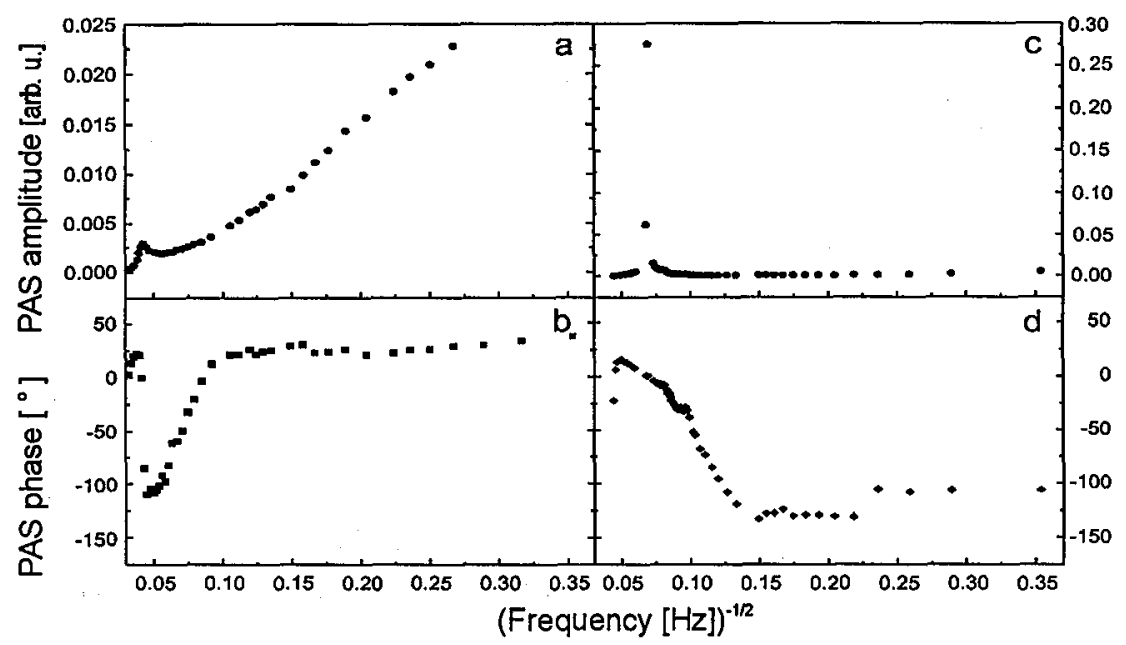

Fig. 2. Photoacoustic signal of olive oil layer (100 $\mu \mathrm{m}$ thickness) spread on the water $(a, b)$ and cooper (c, d) surface; $(a, c)$ amplitude, $(b, d)$ phase of PA signal dependences.

The PA signal resulting from the olive layer deposited onto the water surface is significantly different from the one originating from the homogeneous sample placed on the solid base. The observed amplitude and phase changes of PA signal dependences for oil layers spread on water surface as shown in Fig. 2a and b versus the thermal diffusion length (related via Eq. (1) to the modulation frequency of light) suggest the presence of three separate sublayers differentiated in their thermal parameters. The frequencies, for which abrupt amplitude and phase changes are observed, correspond to the layer thickness of about 9 and $13 \mu \mathrm{m}$ (the layer of about $4 \mu \mathrm{m}$ thick).

A single maximum in the signal amplitude plot from Fig. 2c, for oil layer spread on the copper surface is related to the thermal properties of the solid support. The phase plot goes on regularly and monotonically that is characteristic of uniform layers in Fig. 2d. The same results were also obtained, for oil layers placed onto the copper surfaces in various ways. Consequently, it appeared that the procedure of oil spreading has no effect on the oil-supporting surface interaction phenomenon. 
The results obtained for amplitude and phase plots of the signal with olive oil layers of $100 \mu \mathrm{m}$ thickness deposited on the surfaces of water and copper differ from each other, and evidently depend on a kind of supporting. Differences observed in the amplitude dependences are related to different thermal parameters exhibited by the "used base material", i.e. thermal conductivity $k_{\mathrm{s}}=6.025 \times 10^{-1}$ and $3.975 \times 10^{2} \mathrm{~J} /(\mathrm{s} \cdot \mathrm{m} \cdot \mathrm{K})$ for water and copper, respectively. A metallic support conducts off heat faster from oil layer placed on.

The obtained signal phase plots suggest the presence of the 3-layer structure for a $100 \mu \mathrm{m}$ thick oil layer spread on water as already observed by others [5, 6$]$. As a result of the oil-water interaction at interfaces and in a bulk, oil phase aggregates or micellar-like forms of surfactants can be found if such a surface-active material is present in oil or water at appropriate concentrations (above the critical micellar concentration (CMC)) and temperature (Krafft point).

To perform structure analyses of olive oil layers placed on the water surface, the infrared photoacoustic signal spectra in the range of $4000-1000 \mathrm{~cm}^{-1}$ were taken. Such a range is characteristic of $\mathrm{CH}$ stretching vibrations found in the region of 2700-3100 $\mathrm{cm}^{-1}$, and $\mathrm{CH}_{3}$ bending modes in the range of $1325-1480 \mathrm{~cm}^{-1}$.

The PA signal amplitude for the $100 \mu \mathrm{m}$ thickness of olive oil layer spread on the water surface as a function of frequency of modulation in infrared region is presented in Fig. 3. In the Fourier transform infrared photoacoustic spectroscopy studies the following frequency modulations were selected: $418,195,45 \mathrm{~Hz}$. The first one corresponds to the thin surface layer (a few $\mu \mathrm{m}$ in thickness), the second one is related to the layer found in earlier studies $(9-13 \mu \mathrm{m})$, the last one is referred to the lower oil layer. In data analyses, positions of the spectra maximum were assumed, corresponding to the $\mathrm{CH}$ bonds of interest for us.

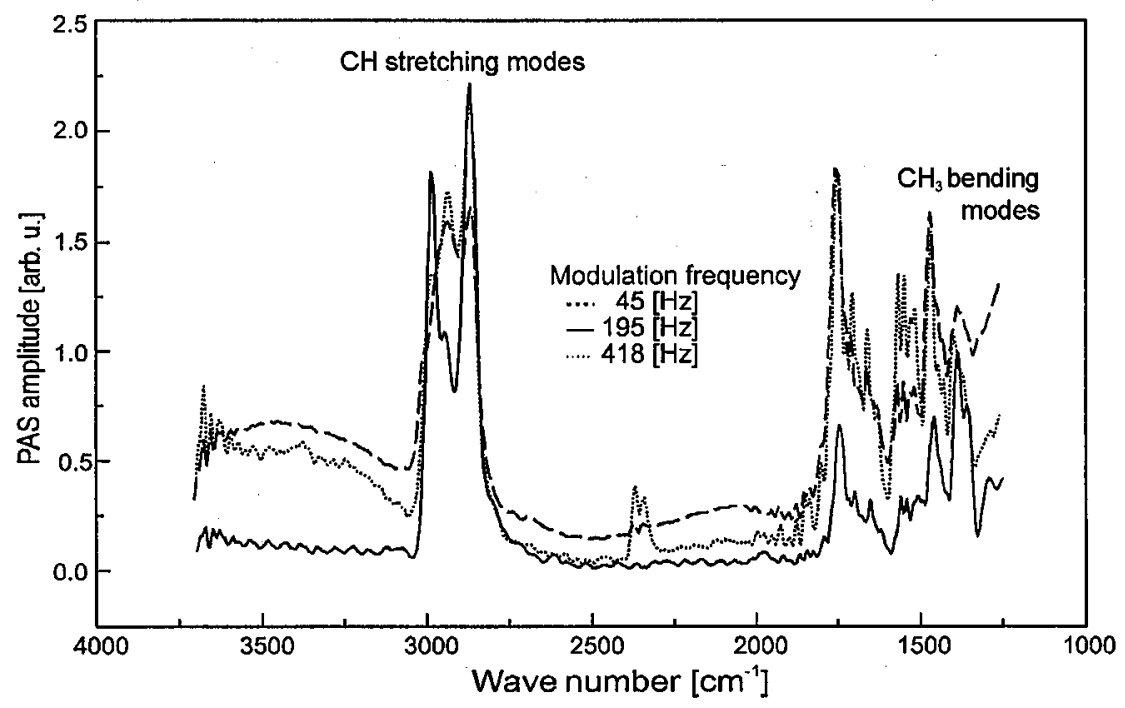

Fig. 3. Photoacoustic spectra of $100 \mu \mathrm{m}$ olive oil spread on the water surface in the range of $4000-1000 \mathrm{~cm}^{-1}$ for three frequencies of light modulation: $45,195,418 \mathrm{~Hz}$. 
A shape of the FTIR spectrum apparently depends on the light modulation frequency. A photoacoustic spectrum as a function of modulation frequency for $100 \mu \mathrm{m}$ oil layer at modulation frequency $195 \mathrm{~Hz}$ is similar to the spectrum for a pure bulk oil (homogeneous layer). This frequency is related to the layer found in earlier studies (a thickness of oil layers of about $4 \mu \mathrm{m}$, situated at a depth of $9-13 \mu \mathrm{m}$ ). Similar in shape spectra were obtained in earlier studies for a $25 \mu \mathrm{m}$ oil layer thickness spread on water $[4,6]$. The modulation frequency does not affect the spectrum shape for $25 \mu \mathrm{m}$ oil layer for all used frequencies. A distribution of the spectrum into bands corresponding to the oscillation vibration of subsequent functional groups under inspection seems to be the same for the studied sample [4].

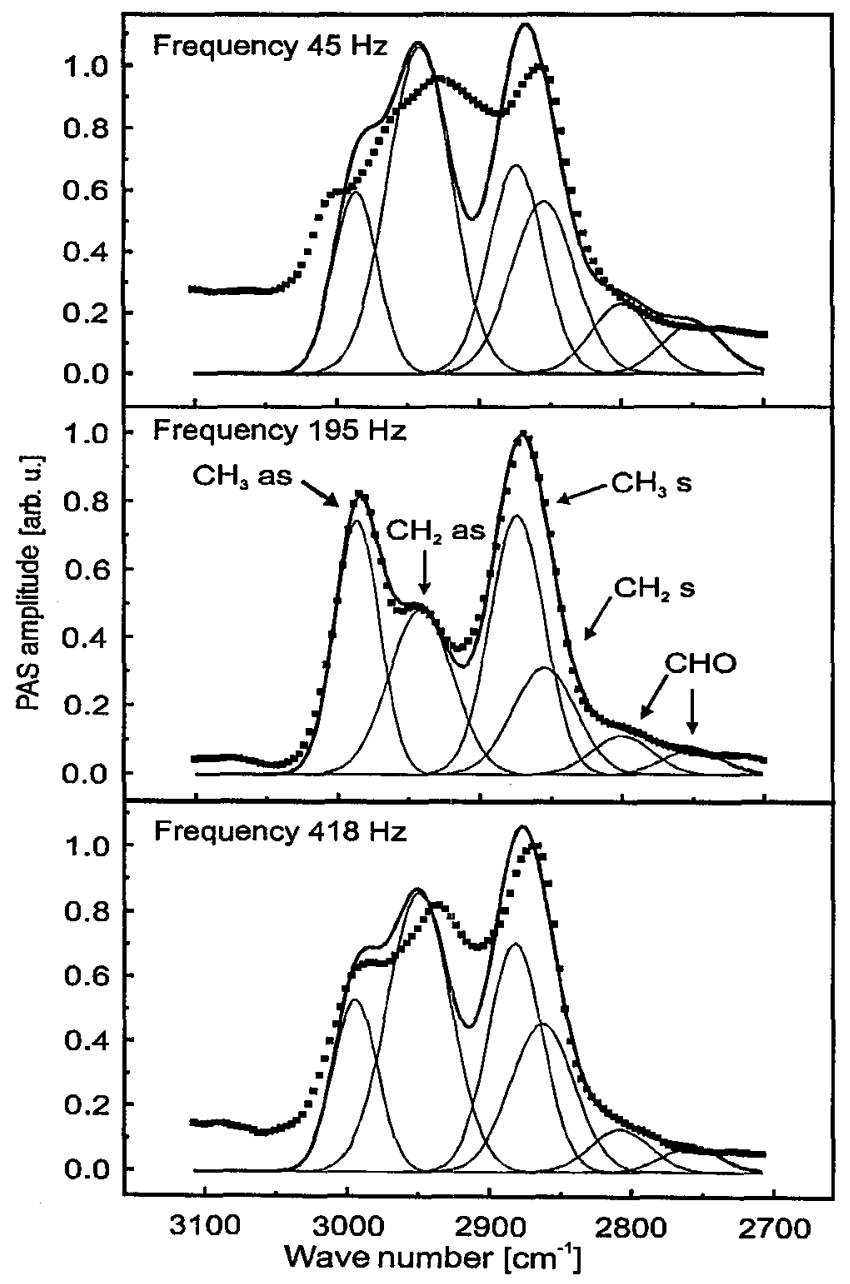

Fig. 4. Photoacoustic spectra of $100 \mu \mathrm{m}$ olive oil spread on the water surface in the range of $3100-2700 \mathrm{~cm}^{-1}$ for three frequencies of light modulation: $45,195,418 \mathrm{~Hz}$ and its Gaussian fitting plots; as - asymmetric, s - symmetric stretching modes. 
Shapes of the spectra of two remaining frequencies ( 45 and $418 \mathrm{~Hz}$ ) for $100 \mu \mathrm{m}$ olive oil layer spread on the water surface are different than these for a structurally uniform sample. For these frequencies in the FTIR PAS spectrum the characteristic maximum for water is observed. We are concerned with a water penetration into the oil layer as a result of oil-water interaction phenomenon.

The photoacoustic signal as a function of wave number for a $100 \mu \mathrm{m}$ oil layer in the region of $2700-3100 \mathrm{~cm}^{-1}$ at three different modulation frequencies is depicted in Fig. 4. While analyzing the obtained spectra in the selected range, a particular position of the maxima and half-widths of the spectral lines corresponding to $\mathrm{CH}$ group bonds was adopted after literature data [9]. The maxima at 2987 and $2942 \mathrm{~cm}^{-1}$ are said to be attributed to the asymmetric oscillation modes of $\mathrm{CH}_{3}$ and $\mathrm{CH}_{2}$ groups, respectively. The maxima at 2874 and $2855 \mathrm{~cm}^{-1}$ were classified as responsible for symmetric vibrations of $\mathrm{CH}_{3}$ and $\mathrm{CH}_{2}$ groups. Two spectral bonds at 2750 and $2800 \mathrm{~cm}^{-1}$ correspond to the weak bands of $\mathrm{CHO}$ group.

Shapes of the spectra of $100 \mu \mathrm{m}$ olive oil layer spread on the water surface for three frequencies differ from each other. That is an evidence of the structural changes within the layer of $100 \mu \mathrm{m}$. A separation procedure of the spectra into the bands related to the corresponding oscillation modes does not lead to satisfactory results. A shift of the maximum related to $\mathrm{CH}_{2}$ groups to the longer wavelength part of the spectrum is observed. It means that the chemical structure change of oil in contact with water took place.

An intensity ratio of the asymmetric to the symmetric $\mathrm{CH}_{2}$ stretching modes can be considered as a measure of the degree of order of the alkyl chains. Its higher value stands for a higher degree of order of the system. The values of the ratio of intensities of the asymmetric to symmetric $\mathrm{CH}_{2}$ group stretching modes are collected in Table I. The ratio of intensities of $\mathrm{CH}_{2}$ asymmetric to $\mathrm{CH}_{2}$ symmetric modes which is related to the organization order does not change within the experimental error for a homogeneous oil layer. For a $100 \mu \mathrm{m}$ thick oil layer, the degree of order increases, and only for the $195 \mathrm{~Hz}$ frequency, corresponding to the distinguished layer in the studies, is equal within the measuring error to the order degree of uniformly structured layer. In the upper and lower layers, where the organization order grows, more organized structures are present. There are

\section{TABLE I}

The ratio of the photoacoustic signal intensity of the asymmetric to symmetric $\mathrm{CH}_{2}$ stretching modes.

\begin{tabular}{c|c|c}
\hline \hline $\begin{array}{c}\text { Modulation frequency } \\
{[\mathrm{Hz}]}\end{array}$ & \multicolumn{2}{|c}{ Ratio of PA signal intensity } \\
& $25 \mu$ m layer & $100 \mu$ m layer \\
\hline 45 & $1.35 \pm 0.10$ & $1.9 \pm 0.5$ \\
195 & $1.42 \pm 0.10$ & $1.5 \pm 0.1$ \\
418 & $1.26 \pm 0.07$ & $1.9 \pm 0.3$
\end{tabular}




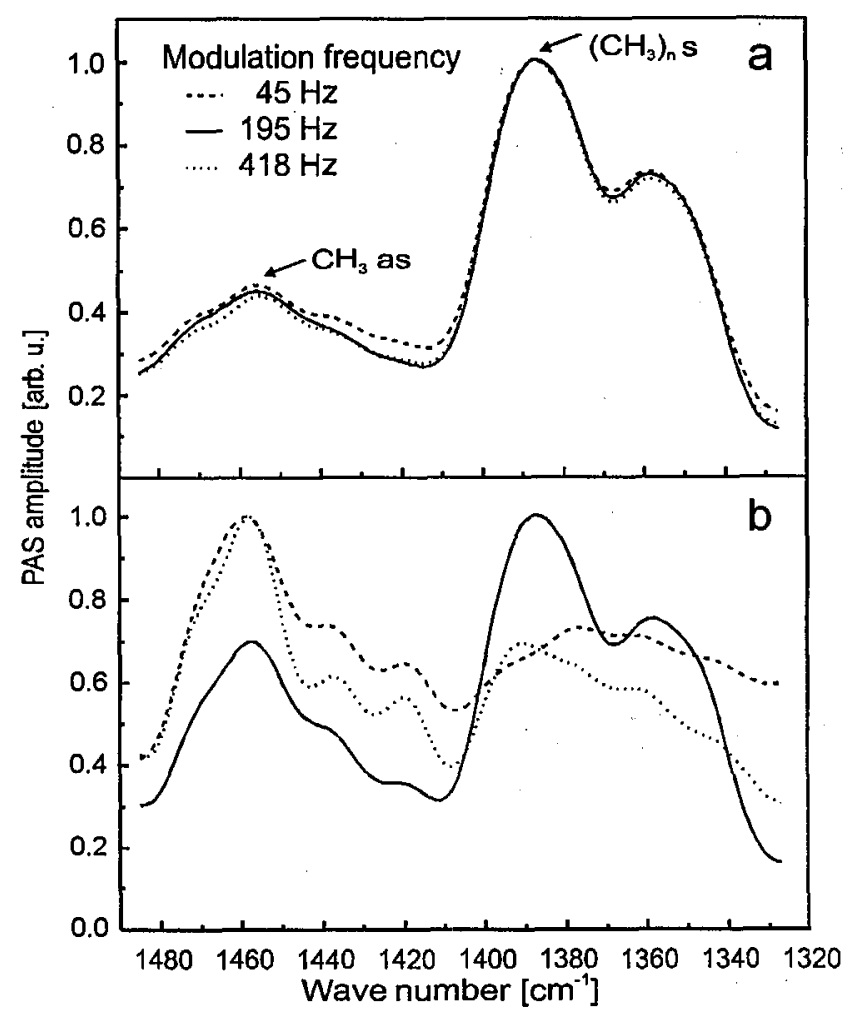

Fig. 5. Photoacoustic spectra of homogeneous layer $-25 \mu \mathrm{m}$ (a) and $100 \mu \mathrm{m}$ (b) olive oil layer spread on the water surface in the range of $1480-1320 \mathrm{~cm}^{-1}$ for three frequencies of light modulation: 45, 195, $418 \mathrm{~Hz}$; as - asymmetric, s - symmetric bending modes.

no longer "pure" $\mathrm{CH}_{2}$ and $\mathrm{CH}_{3}$ group vibration modes as a result of structural changes induced by the oil-water interaction. Probably, micellar structures are formed in the interaction between alkyl chains and water molecules $[5,10]$.

TABLE II

The ratio of the photoacoustic signal intensity (asymmetric $\mathrm{CH}_{3}$ bending vibration band to the symmetric $-\mathrm{C}\left(\mathrm{CH}_{3}\right)_{n}$ bending modes).

\begin{tabular}{c|c|c}
\hline \hline $\begin{array}{c}\text { Modulation frequency } \\
{[\mathrm{Hz}]}\end{array}$ & \multicolumn{2}{|c}{ Ratio of PA signal intensity } \\
$25 \mu \mathrm{m}$ layer & $100 \mu \mathrm{m}$ layer \\
\hline 45 & 0.46 & 1.37 \\
195 & 0.46 & 0.71 \\
418 & 0.46 & 1.46
\end{tabular}


To confirm the occurrence of layered structures the wavelength range of $1325-1485 \mathrm{~cm}^{-1}$ was also analyzed as being characteristic of bending vibration modes of $\mathrm{CH}_{3}$ groups situated at the ends of alkyl chains. In Fig. 5 , such a vibration range is represented for two oil layer thicknesses $25 \mu \mathrm{m}$ (a) and $100 \mu \mathrm{m}$ (b) and three above-mentioned modulation frequencies. According to the literature data [9], the spectral maxima in the range of $1470-1440 \mathrm{~cm}^{-1}$ correspond to the bending asymmetric vibration modes of $\mathrm{CH}_{3}$ groups, whereas the maxima related to symmetric bond $-\mathrm{C}\left(\mathrm{CH}_{3}\right)_{n}$ oscillations lie in the range of $1365-1395 \mathrm{~cm}^{-1}$.

A ratio of the spectral maxima, the one corresponding to the asymmetric bending vibration modes of a $\mathrm{CH}_{3}$ group to that reflecting symmetric vibration modes of a $-\mathrm{C}\left(\mathrm{CH}_{3}\right)_{n}$ group, does not change in the uniform layer (a thickness of $25 \mu \mathrm{m}$ ). For a thicker oil layer (of $100 \mu \mathrm{m}$ thick) the degree of order, similarly like for $\mathrm{CH}_{3}$ groups, depends on the light modulation frequency (see Table II).

\section{Conclusions}

Photoacoustic spectroscopy turned out to be a useful method in structural studies of thin oil layers spread onto the water and copper surfaces.

The photoacoustic signal amplitude and phase dependences versus the exiting light modulation frequency allows the thickness of the sublayers differing from each other in their thermal parameters (thermal conductivity for instant) to be determined.

Oil layers spread on metallic copper surfaces appeared to be uniform in structure without any evidence of substructure formation that is opposite to the oil-water layered systems. The adopted in spreading of oil layers procedures (direct or from an ethyl ether oil solution) had no effect on the structure of oil layer formed neither on water nor on copper surfaces.

An FTIR PAS technique allowed us to correlate the thermal parameters changes observed for an oil layer of $100 \mu \mathrm{m}$ deposited on water, already detected using a classic PAS method, with the structural modifications of the oil studied. Such a molecular organization variability is related to the water molecule penetration into the oil layer. The photoacoustic spectra in their bands responsible for absorption of $\mathrm{CH}_{2}$ and $\mathrm{C}\left(\mathrm{CH}_{3}\right)_{n}$ groups situated in the ends of alkyl chains exhibited signatures corresponding to the modification of the chain organization order due to the interaction with water.

The obtained results suggest a 3-layer vertically separated structural form of the studied oil layer where more organized micellar structures are present in the upper and bottom sublayers. Formation of clathrate (crystal-like structures) and of very fine microemulsions (thermodynamically stable oil-water-surfactant systems) is a common phenomenon in the presence of surface-active substances present as impurities in oil and water especially effective close to the interfacial regions (air-water, air-oil or oil-water). A detailed study of such structures awaits more supplementary data on surfactant physics and chemistry. 


\section{Acknowledgment}

The study was supported in the framework of scientific activity of the University of Gdańsk (BW/5200-5-0306-0 and DS/5200-40024-8).

\section{References}

[1] Micelles, Membranes, Microemulsions and Monolayers, Eds. W.M. Gelbart, A. Ben-Shaul, D. Roux, Springer-Verlag, New York 1994.

[2] R.T. Morrison, R.N. Boyd, Organic Chemistry, PWN, Warszawa 1998 (in Polish).

[3] L. Hernquist, in: Crystallization and Polymorfism of Fatty Acids, Eds. N. Garti, K. Sato, Marcel Dekker Inc., New York 1988, p. 97.

[4] I. Pawelska, J. Szurkowski, in: Proc. 28th Winter School on Molecular and Quantum Acoustics and 4th Workshop on Photoacoustic and Photothermics, Ustroń 1999, Eds. T. Błachowicz, J. Bodzenta, Polish Acoustical Society, Gliwice 1999 , p. 141.

[5] S.J. Pogorzelski, J. Szurkowski, A. Śliwiński, Arch. Acoust. 24, 319 (1999).

[6] J. Szurkowski, S. Wartewig, Instrumentation Science and Technology 27, 311 (1999).

[7] J. Szurkowski, S. Wartewig, in: Proc. X Int. Conf. on Photoacoustic and Photothermal Phenomena, Rome 1998, Eds. F. Scudieri, M. Bertolotti, American Institute of Physics, New York 1999, p. 618.

[8] M. Wegener, R. Neubert, W. Rettig, S. Wartewig, Int. J. Pharmaceutics 128, 203 (1996).

[9] D. Lin-Vien, N.B. Colthup, W.G. Fateley, J.G. Grasselli, Infrared and Raman Characteristic Frequencies of Organic Molecules, Academic Press Inc., San Diego 1991.

[10] W.L. Hinze, J. Am. Chem. Soc. 1,1 (1987). 